\title{
Reconstruction of Fractional Quantum Hall Edges
}

\author{
Xin Wan ${ }^{1}$, Kun Yang ${ }^{1}$, and E. H. Rezayi ${ }^{2}$ \\ ${ }^{1}$ National High Magnetic Field Laboratory and Department of Physics, Florida State University, Tallahassee, Florida 32306 \\ ${ }^{2}$ Department of Physics, California State University, Los Angeles, California 90032
}

(March 31, 2021)

\begin{abstract}
We study the interplay of interaction, confining potential and effects of finite temperature at the edge of a quantum Hall liquid. Our exact diagonalization calculation indicates that edge reconstruction occurs in the fractional quantum Hall regime for a variety of confining potential, including ones that correspond to a "sharp" edge. Our finite temperature Hartree-Fock calculation for integer quantum Hall edges indicates that reconstruction is suppressed above certain temperature. We discuss the implication of our results on recent edge tunneling and microwave absorption experiments.
\end{abstract}

73.40.Hm, 71.10.Pm

The physics of the quantum Hall edge states is important for a number of reasons. When the system is in a quantum Hall state, the bulk is incompressible and the edge carries the dissipationless current. In addition, the edge states also provide a unique arena to study correlated electrons in one-dimension, where the Fermi liquid theory breaks down. Indeed, the edge electrons have been argued [1] to form the so-called chiral Luttinger liquid (CLL) in this case, with many properties determined solely by the robust bulk topological order of the system, and independent of the details of the edge potential. For example it was predicted [1] that in the tunneling between a Fermi liquid and a quantum Hall edge, the current-voltage relation follows a power-law $I \sim V^{\alpha}$, with universal exponents $\alpha$ for simple edges like that of bulk filling $\nu=1 / 3$. While such power-law behavior has been observed in experiments indicating non-Fermi liquid behavior [2], detailed studies of the bulk filling factor as well as sample dependence of $\alpha$ have found important differences from the predictions of CLL theory [3 5 ].

In the CLL theory [1], one assumes that there is a single boundary separating the incompressible quantum Hall liquid from the electron vacuum, and for simple edges like those of $\nu=1$ and $\nu=1 / 3$, the only low-energy excitations are the shape fluctuations of the boundary, which form a single branch of chiral bosons. On the other hand it is known that for integer quantum Hall edges, the competition between electron-electron Coulomb interaction and edge confining potential can lead to edge reconstruction [6,7], when the edge confining potential is smooth enough. At the reconstructed edge, the electron density oscillates and there are more than one chiral boson modes, which do not propagate in the same direction [7]. Thus edge reconstruction can change the physics at the edge qualitatively.

Motivated by the edge tunneling puzzle, as well as a recent microwave absorption measurement in which the presence of artificial edge channels leads to absorption enhancement [8], we examine in this work the possibility of edge reconstruction when the bulk filling is frac- tional. We find numerical evidence from exact diagonalization studies suggesting that edge reconstruction can occur when the bulk filling is fractional as well, for a variety of edge confining potentials (including those we believe properly describe a sharp cleaved edge), and filling factors. We also study the effect of finite temperature on edge reconstruction using Hartree-Fock approximation on a $\nu=1$ edge, where we find thermal fluctuations tend to suppress edge reconstruction. We argue that our results are highly relevant to and shed considerable light on both the edge tunneling and the microwave absorption experiments. We also comment on the differences and similarity between our results and those of a recent numerical study of the $\nu=1 / 3$ edge 9 .

Exact Diagonalization Study - In this work we diagonalize the following Hamiltonian exactly, which describes electrons confined to the lowest Landau level, using the symmetric gauge:

$$
H=\frac{1}{2} \sum_{m n l} V_{m n}^{l} c_{m+l}^{\dagger} c_{n}^{\dagger} c_{n+l} c_{m}+\sum_{m} U_{m} c_{m}^{\dagger} c_{m},
$$

where $c_{m}^{\dagger}$ is the electron creation operator for the lowest Landau level single electron state with angular momentum $m, V_{m n}^{l}$ is the matrix element of Coulomb interaction, and $U_{m}$ is the matrix element of a rotationally invariant confining potential due to the positive background charge. We have studied systems with $N=4$ to 12 electrons, at a number of filling factors. Figure 11(a) shows the ground state energy 10 $E_{0}(M)$ of a given subspace with a fixed total angular momentum $M$ as a function of $M$, for $N=6$ electrons. The confining potential $U_{m}$ is calculated from a disc of uniformly distributed positive background charge placed at a distance $d$ from the electron layer, and the radius of the disc $a$ is chosen such that the disc encloses 18 flux quanta. The charge of the disc is always chosen to be the same as that of the electrons so that the system is neutral. Furthermore, we restrict the electrons to 18 orbitals, from $m=0$ to $m_{\max }=17$; we believe this properly describes a sharp cleaved edge where the electrons cannot move beyond 
where the confining charge ends. Thus in this case our model describes a 6 electron system at $\nu=1 / 3$, confined by both the background charge and a sharp edge [11]. Fig. 2 is an illustration of our model. As we change the confining potential by increasing $d$ from $d=0.1$ to 5 , in units of the magnetic length $l_{B}$, the total angular momentum $M$ of the global ground state increases from $M=45$ to 65 . We note that the Laughlin state for 6 electrons has total angular momentum $M=45$. This indicates that while the global ground state of the system is well described by the Laughlin state for $d<1.6$, edge reconstruction occurs for $d \geq 1.6$, as indicated by the increase of $M$ [6,7].

The presence of edge reconstruction can also be seen in the electron density profile near the edge, as we plot in Fig. Il(b)-(e), and compare them with the Laughlin state. Despite the Coulomb interaction and the confining potential, the electron density for $d=0.1$ closely resembles the Laughlin state, with slightly stronger oscillation which is characteristic of the Coulomb interaction. Our calculations show that the critical $d$ above which the total angular momentum $M$ of the global ground state differs from the Laughlin state is between 1.5 and 1.6 for this system. The corresponding electron density for $d=1.6$ (Fig. 1 1 (c)) shows much larger oscillation than that of the Laughlin state. The critical $d$ fluctuates around $d_{c}=1.6 \pm 0.1$ for system as large as 9 electrons in 27 orbitals (Fig. 11(a) inset). It is thus reasonable to expect that the critical $d$ for edge reconstruction at $\nu=1 / 3$ lies between 1 and $2 l_{B}$ in the thermodynamic limit, considerably smaller than the typical $d$ in experiments $\left(5-10 l_{B}\right)$. The change of $M$ at $d=d_{c}$ increases with $N$ as expected. As $d$ further increases, the oscillation becomes stronger so that the electron density gradually separates into an edge piece and a center piece. The edge piece is almost detached from the center piece for $d=5.0$, providing further evidence of edge reconstruction at fractional fillings.

Edge reconstruction is the result of competition between Coulomb interaction and confining potential [6.7. In our model the confining potential is controled by $d$; the larger $d$ is, the weaker the confinement is at the edge due to the backgroud charge, thus edge reconstruction occurs above certain $d_{c}$. Another way to understand this fact is that without edge reconstruction, the electron density is approximately uniform all the way to the edge, thus the electron gas layer and the background charge layer form a capacitor (see Fig. 22). In such a capacitor the electrostatic potential is a constant in the bulk of the electron layer, but a gradient (or fringe electric field with in-plane component) develops at the edge, which tends to pull the electrons toward the edge. The larger $d$ is, the larger the area is affected by this fringe field, and eventually reconstruction occurs. Since in the systems we study the linear size of the system is much larger than $d_{c}$, the finite size effects are weak (as also indicated by the weak dependence of $d_{c}$ on $N$ ); a detailed analysis of the finite
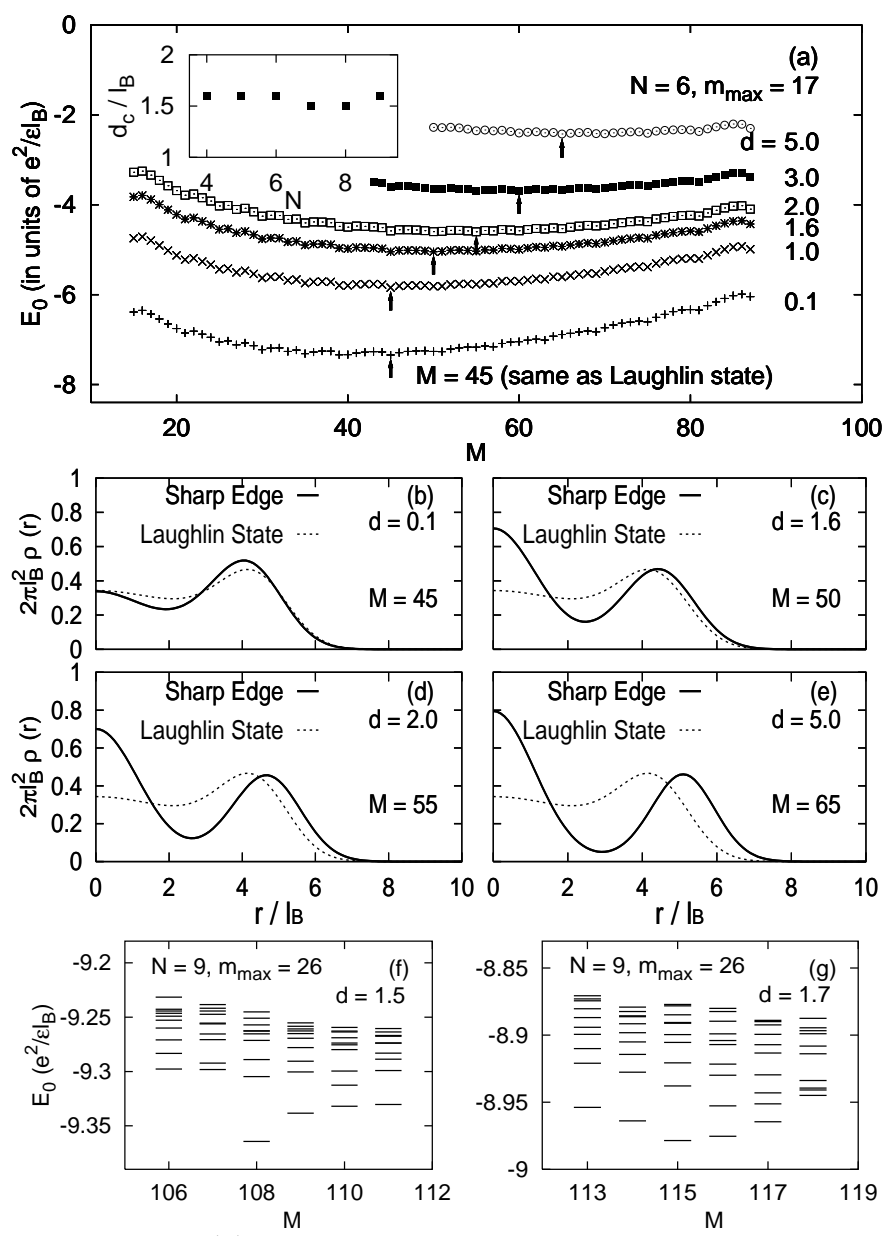

FIG. 1. (a) Ground state energy of each total angular momentum $(M)$ subspace as a function of $M$ for 6 electrons in 18 orbitals, with the background charge uniformly distributed on a disc above the electron layer with a distance of $d$ from 0.1 to $5 l_{B}$. The global ground state for each $d$ is indicated by an arrow below the corresponding curve. The inset shows the critical $d$ above which $M$ of the global ground state differs from the corresponding Laughlin state as a function of the number of electrons $N$ at $\nu=1 / 3$. The electron densities $\rho(r)$ of the global ground state for 6 electrons are compared with that of the Laughlin state (dotted lines) for (b) $d=0.1$, (c) $d=1.6$, (d) $d=2.0$ and (e) $d=5.0$, in units of $l_{B}$. The low-lying states near the global ground state are shown for $N=9$ electrons for (f) $d=1.5$ (before edge reconstruction) and (g) $d=1.7$ (after edge reconstruction).

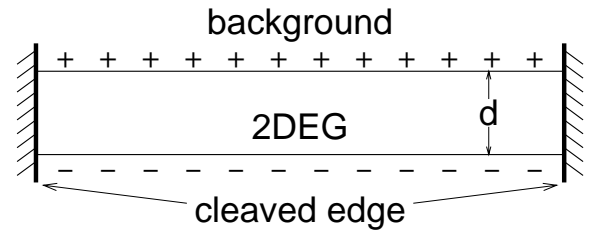

FIG. 2. Side view of the electron gas layer and the background charge layer with a sharp cleaved edge. 


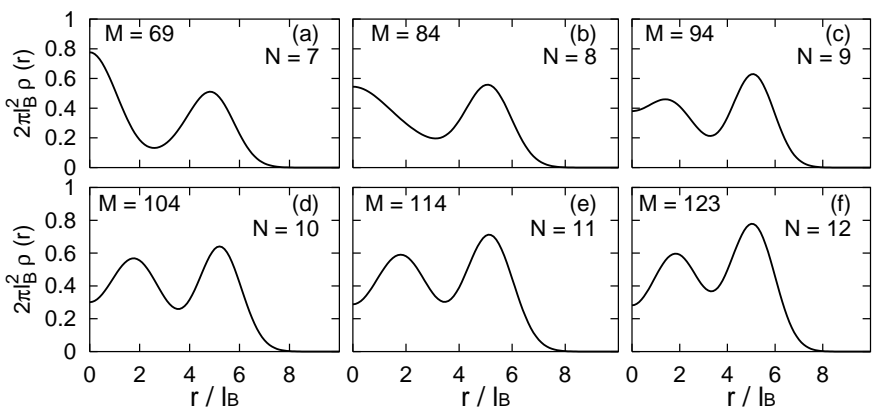

FIG. 3. The electron density $\rho(r)$ of the lowest energy state for $N=7$ to 12 electrons in 18 orbitals with uniform background charge, distributed $d=3 l_{B}$ above the electron layer.

size effects will be presented elsewhere. We also note an important difference bewteen the integer and fractional edge reconstruction; in the former the presence of a sharp boundary alone eliminates the possibility of reconstruction due to Pauli principle; it is no longer the case for the fractional edge.

Besides the single chiral mode predicted by the CLL theory [1], additional edge modes are expected for reconstructed edges [7]. We find evidence for this in the spectra. Figs. 1(f) and $1(\mathrm{~g})$ are the low-energy spectra for $N=9$ systems at $\nu=1 / 3$, before and after edge reconstruction. We find before reconstruction low-lying states only exist for $M>M_{\text {ground }}=108$, consistent with the presence of a single chiral mode; while after reconstruction there are low-lying states with $M$ both above and below $M_{\text {ground }}=115$, indicating that there are modes propagating in both directions.

Indication of edge reconstruction persists when we increase the average filling factor from $\nu=1 / 3$ to $2 / 3$. Figure 3(a)-(f) show the electron densities of the global ground states for up to 12 electrons in 18 orbitals. The background charge is confined within the same radius $a$ as above, at a distance of $3 l_{B}$ above the electron layer, with appropriate charge density to ensure charge neutrality. In all cases, we find indication of edge reconstruction as for $N=6$. As $N$ increases, the electron density in the edge piece increases accordingly, while its peak location remains almost unchanged. It should be noted however, that in these cases the filling factor for the bulk is less well-defined, as the "bulk" area is too small and the electron density still oscillates at $r=0$. Nevertheless, we believe that the tendency for electron density to reconstruct at the edge is a generic feature for appropriately confined electrons at any fractional filling.

While a sharp cutoff (at $m_{\max }$ ) where the background charge ends properly describes a cleaved edge, it is not present for other edges. Not surprisingly, we find that removing this sharp cutoff further favor edge reconstruction. Evidence for edge reconstruction in a system with a smooth edge will be presented elsewhere.

Hartree-Fock Calculation - We now turn to a dis-
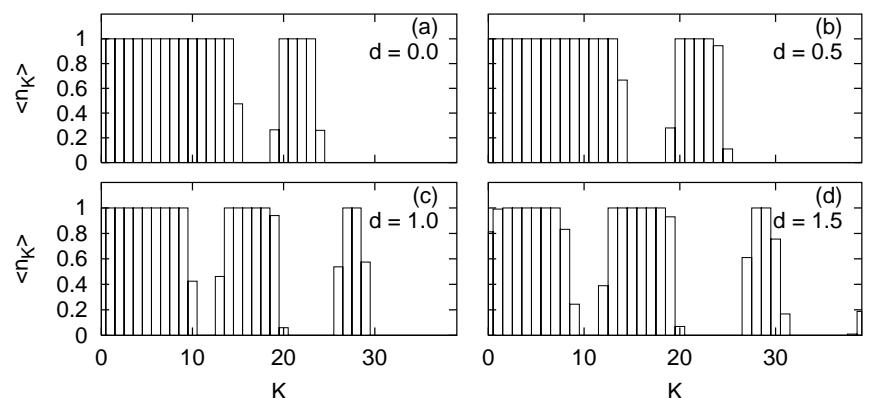

FIG. 4. Occupation numbers for background charge with $W=10 l_{B}$ and different $d$ from 0 to $1.5 l_{B}$ calculated within finite- $T$ Hartree-Fock approximation at $T=0.001 e^{2} / \epsilon l_{B}$.

cussion of the effect of a finite temperature $T$ on a reconstructed edge. To this end we will use a finitetemperature Hartree-Fock (HF) approximation to study the $\nu=1$ edge. Laying the background charge right on top of the electron layer, Chamon and Wen [7] demonstrated that a lump of electron density can move $\sim 2 l_{B}$ away from the $\nu=1$ bulk for smooth enough background charge distribution. In their model the smoothness of the edge potential is controlled by the width $W$ over which the positive background charge density decreases from the bulk value to zero. They have also shown that the results of HF approximation agree well with those of exact diagonalization at $T=0$. Here we use the finitetemperature Hartree-Fock approximation for the interacting electron system, with cylindrical geometry similar to the one studied by Chamon and Wen [7], with an extra parameter $d$ which is the distance the background charge lies above the electron gas. In Fig. [1(a)-(d) we show results of the average electron occupation numbers at very low temperature $T=0.001$, in units of $e^{2} / \epsilon l_{B}$, for 20 edge electrons in 40 single particle states on a strip of length $L=20 l_{B}$ with periodic boundary conditions, background charge $W=10 l_{B}$, and $d$ varying from $d=0$ to $1.5 l_{B}$. We find that the edge reconstruction pattern depends on the detail of the edge confining potential. States with linear momentum $K<0$ ("bulk") and $K \geq 40$ are assumed to be inert, with occupation number $n_{K}$ fixed to be 1 and 0 , respectively. Fig. 1 (a) essentially reproduces the $T=0$ results by Chamon and Wen. As $d$ increases (so that the confining potential gets even smoother), the detached piece becomes wider and an extra piece appears for $d=1.0$ and 1.5. The size of the reconstructed region increases rapidly with $d$, and goes beyond what we can handle numerically for $d \geq 2.0 l_{B}$.

The edge reconstruction disappears as we increase the temperature. Figure 5(a)-(d) show the occupation numbers for background charge with $W=10 l_{B}$ and $d=0$ (Fig. 国(a)) at higher temperatures. We double the number of active single particle states to 80 , as well as the number of edge electrons to $N=40$, to ensure that the bulk states $(K<0)$ are fully occupied (within $99 \%$ percent), a posteriori condition for separating the elec- 

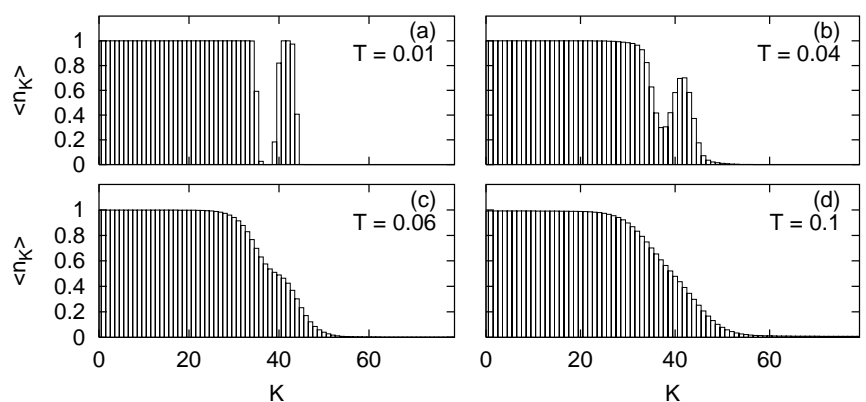

FIG. 5. Occupation numbers for background charge with $W=10 l_{B}$ and $d=0$ at different $T$ within $\mathrm{HF}$ approximation.

trons into "edge" and "bulk" ones. The length of the strip remains at $L=20 l_{B}$. The detached edge piece at $T \leq 0.01$ mixes with the bulk at $T=0.04$, although a well-defined peak can still be resolved. The peak evolves to a shoulder at $T=0.06$, disappearing before $T$ reaches 0.1 , where the occupation number decreases smoothly from 1 to 0 , mirroring the smoothed background charge profile. Although extra pieces can emerge for finite $d$, we find that all edge pieces merge with the bulk and disappear at $T \sim 0.05$. We thus conclude that, within the Hartree-Fock approximation, edge reconstruction disappears above $T \sim 0.05 e^{2} / \epsilon l_{B}$ at $\nu=1$. We have obtained a very similar temperature scale above which edge reconstruction disappears for the $\nu=1 / 3$ edge, using results of exact diagonalization (that generates all eigenstates), in systems with up to 7 electrons; details will be presented elsewhere.

Discussions — When the edge reconstructs, there are multiple edge modes even for the simplest quantum Hall states like $\nu=1$ and $\nu=1 / 3$ [7]. In particular, these modes do not propagate in the same direction, and as a consequence the tunneling exponent $\alpha$ depends on the interaction among these modes and is thus no longer universal, even for these bulk fillings. Thus the edge reconstruction can very well be the reason why $\alpha$ is always found to be slightly less than the CLL prediction of $\alpha=3$ at $\nu=1 / 3$, and why it varies continuously with bulk filling and other sample parameters, while CLL predicts plateau behavior [12]. On the other hand experiments find [3] a very simple (but approximate) relation $\alpha \sim 1 / \nu$ (see, however, Refs. [2.5]), for which we do not have an explanation here. Nevertheless we believe edge reconstruction is likely to be an important piece of the edge tunneling puzzle, and should be included as part of the complete theory for the fractional quantum Hall edge.

In a recent microwave absorption experiment [8], it was found that the conductivity at microwave frequency is strongly enhanced in samples with antidots in the fractional quantum Hall regime at very low temperatures, while no such enhancement was found in similar samples with no antidots. It is thus natural to associate the enhancement with the edge channels of the antidots. On the other hand, the dots are so small that it is easy to convince oneself that the frequency of the edge magneto plasma (EMP) is much higher than the microwave frequency used in the experiments (by about an order of magnitude). It thus appears unlikely that the enhancement is due to the microwave frequency approaching that of EMP. On the other hand, a reconstructed edge can support softer acoustic modes, which may well be accessible in the experimental frequency range [13]. Another important experimental finding is that the enhancement disappears above temperature $T^{*} \sim 0.5 K$. This is qualitatively consistent with our finding that edge reconstruction disappears at higher $T$. While the Hartree-Fock estimate of this temperature scale: $T \sim 0.05 e^{2} / \epsilon l_{B} \sim 6 K$, is higher than $T^{*}$, we note that our calculations tend to overestimate this temperature due to finite size effects as well as the fact that we have not included factors such as finite layer thickness, Landau level mixing and disorder not included in our calculations; they all tend to lower this scale. We thus conclude that the results of Ref. \& can be explained as due to edge reconstruction at low temperatures.

In a recent study, Tsiper and Goldman [9] found electron density oscillation near the edge of a electron droplet at $\nu=1 / 3$, which they interpret as formation of edge density wave. In their study the confining potential due to the background charge is totally neglected, while we believe it is extremely important for a proper understanding of the physics at the edge. Moreover, they have focused only on states that have the same quantum number as the Laughlin state; as we demonstrated this is often not the case for the global ground state, a fact which is precisely the indication of edge reconstruction. On the other hand the edge density oscillation they find is quite similar to those of reconstructed edges reported here.

The authors thank Lloyd Engel and Dan Tsui for stimulating discussions that motivated the present work and useful comments on the manuscript, and Claudio Chamon, Allan MacDonald, and Shivaji Sondhi for helpful conversations. EHR gratefully acknowledges the hospitality of the NHMFL where part of this work was performed. This work was supported by NSF grants DMR9971541 (XW and KY), DMR-0086191 (EHR), the State of Florida (XW), and the Sloan Foundation (KY).

[1] X.-G. Wen, Int. J. Mod. Phys. B 6, 1711 (1992).

[2] A. M. Chang, L. N. Pfeiffer, and K. W. West, Phys. Rev. Lett. 77, 2538 (1996).

[3] M. Grayson et al., Phys. Rev. Lett. 80, 1062 (1998).

[4] A. M. Chang et al., Phys. Rev. Lett. 86, 143 (2001).

[5] M. Hilke et al., Phys. Rev. Lett. 87, 186806 (2001).

[6] A. H. MacDonald, S. R. E. Yang, and M. D. Johnson, 
Aus. J. Phys. 46, 345 (1993).

[7] C. de C. Chamon and X.-G. Wen, Phys. Rev. B 49, 8227 (1994).

[8] P. D. Ye, et al., cond-mat/0103127 (to be published in Phys. Rev. B).

[9] E. V. Tsiper and V. J. Goldman, Phys. Rev. B 64, 165311 (2001).

[10] We do not include the self-interaction of the background charge (which is a constant for a given system size) in our definition of the energy.

[11] We note that for the Laughlin state at $\nu=1 / 3$, single electron states are populated only up to $m_{L}=3(N-1)=$ $m_{\max }-2=15$ for $N=6$. We have checked that choosing $m_{\max }$ to be $m_{L}$ instead makes very small differences to $d_{c}$, and the difference shrinks to zero as $N$ increases.

[12] While more recent theories [see, e.g., L. S. Levitov, A. V. Shytov, and B. I. Halperin, Phys. Rev. B 64, 075322 (2001) and references therein] addressed the continuous dependence of $\alpha$ on $\nu$, they all predict $\alpha=3$ at $\nu=1 / 3$ in the absence of edge reconstruction.

[13] We thank S. Sondhi for a useful discussion on this point. 